\title{
ASYMMETRY IN THE PERMANENT PRICE IMPACT OF BLOCK PURCHASES AND SALES: THEORY AND EMPIRICAL EVIDENCE
}

\author{
Alex Frino \\ Vito Mollica \\ Maria Grazia Romano
}


ISBN: 978-88-6197-058-08

ISSN: 1971-3029

Dipartimento di Scienze Economiche e Statistiche Università Degli Studi di Salerno

Via Ponte Don Melillo - 84084; Fisciano (SA) - Italy

Tel + +39-089-96.21.55

Fax +39-089-96.20.49

E-mail dises@unisa.it

Web www.dises.unisa.it 


\title{
ASYMMETRY IN THE PERMANENT PRICE IMPACT OF BLOCK PURCHASES AND SALES: THEORY AND EMPIRICAL EVIDENCE
}

\author{
Alex Frino* $\quad$ Vito Mollica ${ }^{\dagger} \quad$ Maria Grazia Romano ${ }^{\ddagger}$
}

\begin{abstract}
This paper extends previous research which has examined the market impact of large transactions in bull and bear markets by examining the information effects of trades. Previous research has demonstrated that the information effects of buy trades are greater than the information effects of sell trades. We develop a theoretical model which predicts that this difference is greater in bear markets than bull markets, consistent with the (almost counter-intuitive) proposition that buy trades are relatively more informed in bear markets. Using a sample of trades executed on the NYSE in bull and bear market periods, we find evidence consistent with our primary theoretical model.
\end{abstract}

Keywords: block trade, market impact, asymmetry.

JEL classifications: G14, G15.

Acknowledgements: We would like to thank participants in the seminars held at the CSEF, Federico II, University of Sydney and Macquarie University. We especially thank the Securities Industry Research Centre of Asia Pacific for data and technical support. Any errors or omissions are the responsibility of the authors alone.

\section{Introduction}

A large set of literature has examined the price impact of large or block trades in securities markets. ${ }^{1}$ Holthausen, Leftwich and Mayers (1990) are typical. Using a sample of block buy and sell trades executed on the New York Stock Exchange between 1982 and 1984 they demonstrate the following 3 asymmetries in the impact of block purchases and sales. First, that the total price impact of purchases is greater than sales. That is, that the magnitude of the difference between the price at which a block purchase is executed relative to the equilibrium price prior to the trade, is greater than the magnitude of the difference in the price at which a block sale is executed relative to the equilibrium price prior to the trade. Second, they documented an asymmetry in the so-called 'temporary 'price impact of trades. That is, that prices reverse following block sales but continue moving upwards following block purchases. This implies that block sales pay a liquidity premium to trade whereas block purchases do not. Third, they documents that the permanent price impact associated with purchases is greater than sales. That is, that the magnitude of the change in the price of a stock from the equilibrium price prior to a block purchase to the equilibrium price following the purchase, is greater than the magnitude of the change in the price of a stock

\footnotetext{
*University of Sydney, Australia alex. frino@sydney . edu . au

${ }^{\dagger}$ Macquarie Graduate School of Management, Australia, vito.mollica@mgsm. edu . au

${ }^{\ddagger}$ DISES, Via Ponte Don Melillo, 84084, Fisciano (SA), Italy, maromano@unisa . it

${ }^{1}$ These include Kraus and Stoll (1972); Holthausen et. al (1987, 1990); Keim and Madhavan (1996), Aitken and Frino (1996); Chan and Lakonishok, (1993,1995); Gemmill, (1996) and Chiyachantana, Jain, Jiang and Wood (2004)
} 
from the equilibrium price prior to a block sale to the equilibrium price following the sale. This implies that, on average, block purchases convey more information to the market than block sales.

Chiyachantana et. al (2004) extend previous work, by examining the total price impact of trades in bull and bear markets. Using 'packages 'of sell trades and buy trades executed by institutional investors in 37 countries including the USA, they find that the magnitude of the total price impact of buys is greater than the magnitude of the price impact of sells in a bull market period sampled from 1997 and a bear market period sampled from 2001. They conclude that

"our results seem to suggest that due to large trade sizes the institutional trades tend to be affected by market conditions and pay a higher premium for liquidity when they trade on the same side of the market. This finding suggests that the liquidity available to buy (sell) orders is higher in bearish (bullish) markets. This explanation of the asymmetry in price impact is very intuitive and, yet, was not explored in the previous studies because they were all conducted when the market was in a bullish phase.”[p. 871.]

While Chiyachantana et. al (2004) examine the total effects of trades, they do not examine the permanent or information effects of trades. Consequently, we extend the Chiyachantana et. al (2004) analysis by examining the information effects of large or block trades in bull and bear markets. We develop a theoretical model á la Easley and O'Hara (1987) which produces somewhat counter-intuitive predictions of the information effect of block buy trades relative to sell trades in bull and bear markets. We then test the predictions of the model using a sample of data drawn from the bull and bear market periods examined by Chiyachana et.al (2004). To our knowledge, no other study has examined the information effects of block buy and sell trades in bull and bear market conditions.

In our sequential trading model traders are allowed to transact order for block (large) or small quantities. Risk neutral informed traders prefer to trade in blocks at any given prices. Consequently, the market maker sets a wider spread for block traders. However, if the market width is large enough, informed traders place only large orders and small orders are uninformative in equilibrium. ${ }^{2}$ A crucial assumption is that informed traders are averse to short sales. This behavioural assumption gives informed traders a disincentive to sell the asset and can reduce the information content of sell orders. Indeed, we show that, if the market width is large enough, the information effects of block buys is always larger than the information effects of block sells, because of the short sales aversion. Moreoever, in our model, contrarian signals are more valuable than confirming signals. Indeed, traders getting an adverse signal on the true asset value have a larger informational advantage than traders getting a favourable signal if the market is in a bullish phase and lower informational advantage if the market is in a bearish phase. This yields the interesting empirical implication that the difference between the information effects of block purchases and sales is higher in bear markets than in a bull markets.

We analyse a sample of individual transactions (not packages) executed in S\&P 500 stocks during the bull and bear market periods identified by Chiyachantana et. al (2004). Using a method similar to Holthausen et. al (1990) we re-document the asymmetry in the permanent price impact of trades in that the average permanent price impact of buys is greater than the permanent price impact of sells across the entire (bull and bear market)

\footnotetext{
${ }^{2}$ Easley and O'Hara (1987) describe the necessary conditions for the separating equilibrium where informed traders place only large orders.
} 
sample period. Further, we re-document the finding of Chiyachantana et. al (2004) using our sample of individual transactions, and find that the total price impact of buys is greater than sales in a bull market and the total price impact of sells is greater than buys in a bear market. We then proceed to test the main prediction of our theory. Consistent with our theory, we find that the permanent price impact of purchases is greater than the information effects of sales and that this difference is greater in bear markets than bull markets. The remainder of this paper is structured as follows. In section 2 we describe the theoretical model. In section 3 we derive empirical predictions about the asymmetric price impact of block trades in bearish and bullish markets. In section 4 we describe our data and method used to test the theoretical predictions of the model. In section 5 we report the results of our analysis, while section 6 provides a summary and conclusion. All proofs of propositions are in the appendix.

\section{The Model}

\subsection{The Economy}

We consider a sequential trading model analogous to Easley and O'Hara (1987), simplified because there is no event uncertainty.

The market is for a risky asset which is exchanged among a sequence of risk neutral traders and competitive market makers who are responsible for quoting prices. The liquidation value $\widetilde{V}$ of the asset can be low $(\widetilde{V}=\underline{V}=0)$ or high $(\widetilde{V}=\bar{V}=1)$. The ex-ante probability of $\widetilde{V}=\bar{V}$ is $\pi_{0} \in(0,1)$.

Trades occur sequentially, with only one trader allowed to transact at any point in time. The trader whose turn it is to transact may either buy a small or a large quantity, or sell a small or a large quantity, or refrain from trading.

We denote order size using $Q_{S}$ and $Q_{L}$ for small and large orders, respectively (hence $\left.Q_{S}<Q_{L}\right) . A \equiv\left\{S Q_{L}, S Q_{S}, B Q_{S}, B Q_{L}, N T\right\}$ is the traders' action set, with $S Q_{i}$ and $B Q_{i}$ indicating, respectively, a sell and a buy order for quantity $Q_{i}$, with $i=S, L$, and $N T$ indicating no trading.

There are two types of traders: liquidity traders (fraction $1-\mu$ ) and institutional traders (fraction $\mu$ ). We assume that liquidity traders choose to submit large or small sell and buy orders, or to refrain from trading with equal probability $1 / 5$. We denote the probability that an uninformed trader submits a given order as $\gamma \equiv \frac{1-\mu}{5}$.

Institutional traders privately observe a signal $\theta$ correlated with the final asset value. 3 Institutional traders choose the trading strategy that maximizes their profits given the quoted bid and ask prices. However, we assume that, because of restrictions to short sales, institutional traders limit themselves to selling the asset only if it is in their portfolio. ${ }^{4}$ The fraction of informed traders who own the asset and, hence, can sell it is $\delta \in(0 ; 1] .^{5}$

\footnotetext{
${ }^{3}$ All results remain true even if private signals are imperfect, that is, if $\operatorname{Pr}(\underline{\theta} \mid \underline{V})$ and/or $\operatorname{Pr}(\bar{\theta} \mid \bar{V})$ are lower than 1.

${ }^{4}$ Keim and Madhavan (1995) propose the traders aversion to short selling as a possible reason for differences in the information content of trades. They suggest that buy are more informationally motivated than sells because institutional traders can choose between many potential assets to buy, but when they sell, they usually can choose only between assets they already own due to short selling constraints.

${ }^{5}$ We use the terms "institutional traders" and "informed traders" interchangeably.
} 
Before a trader arrives, each market maker sets bid and ask prices at which he is willing to trade each asset quantity.

We denote the probability that the market makers attache to $\bar{V}$ at time $t$ by $\pi_{t}$ and the market makers' expectation at time $t$ is $E_{t}[\widetilde{V}]$. Since $\underline{V}=0$ and $\bar{V}=1, E_{t}[\tilde{V}]=\pi_{t}$. We define the market as bearish when the low is more likely than the high asset value and as bullish when the high is more likely than the low asset value. Hence, a bullish market is characterized by $\pi>1 \backslash 2$ and a bearish market by $\pi<1 \backslash 2$. Finally, the expected asset value of an informed trader is denoted by $E[\widetilde{V} \mid \theta]$ and, since private signals are perfect, it is equal to 1 if $\theta=\bar{\theta}$ and to 0 in the other case.

\subsection{Equilibrium Strategies and Prices}

At the beginning of any trading round $t$, the market maker set her quote prices. We denote the best price schedule at time $t$ by $P_{t}$. Clearly

$$
P_{t}=\left\{B_{L, t}, B_{S, t}, A_{S, t}, A_{L, t}\right\},
$$

where $B_{L, t}$ and $A_{L, t}$ are the bid and the ask prices for the large orders, whilst $B_{S, t}$ and $A_{S, t}$ are the bid and the ask prices for the small orders.

After prices are set, a trader is randomly selected to trade, observes the price schedules and executes her strategy at the best price, or refrains from trading. If he is a liquidity trader, he acts in an ex-ante specified probabilistic way. If he is informed, he chooses the strategy which maximizes her expected profit given the price schedule.

The market maker anticipates the traders' strategies and announces her price schedule. Bertrand competition restricts the market makers to earn zero expected profit from each trade. Hence, the trader arriving at $t$ faces a price schedule which satisfies:

$$
\begin{aligned}
& B_{i, t}=E_{t}\left[\widetilde{V} \mid S Q_{i}\right]=\frac{\operatorname{Pr}\left(S Q_{i} \mid \bar{V}\right) \pi}{\operatorname{Pr}\left(S Q_{i} \mid \bar{V}\right) \pi+\operatorname{Pr}\left(S Q_{i} \mid \underline{V}\right)(1-\pi)} \\
& A_{i, t}=E_{t}\left[\widetilde{V} \mid B Q_{i}\right]=\frac{\operatorname{Pr}\left(B Q_{i} \mid \bar{V}\right) \pi}{\operatorname{Pr}\left(B Q_{i} \mid \bar{V}\right) \pi+\operatorname{Pr}\left(B Q_{i} \mid \underline{V}\right)(1-\pi)},
\end{aligned}
$$

for all $i \in\{S, L\}$.

Since the market makers are imperfectly informed about the liquidation asset value, competitive prices are always between 0 and 1 . If the true asset value is high, institutional traders receive the good signal and buy the asset when they arrive at a market maker. On the other hand, if the true asset value is low, institutional traders receive the bad signal and sell the asset if it is in their portfolio (with probability $\delta$ ) when they arrive at a market maker. It is clear that, since private signals are perfect, the probability of an informed buyer conditional on the low asset value, and the probability of an informed seller conditional on the high asset value, are both zero. This implies that $\operatorname{Pr}\left(B Q_{i} \mid \underline{V}\right)=\operatorname{Pr}\left(S Q_{i} \mid \bar{V}\right)=\gamma$ for both small and large orders.

From Easley and O'Hara (1987) we know that, depending on the parameters of the model, two outcomes may prevail on each side of the market. If informed traders prefer to trade only a large quantity, they are separated from small liquidity traders and a separating equilibrium arises. If informed traders submit either small or large orders with strictly positive probability, a pooling equilibrium occurs. 
We first examine the market in the separating equilibrium. In this market, the competitive price schedule, $P^{s e}=\left\{B_{L}^{s e}, B_{S}^{s e}, A_{S}^{s e}, A_{L}^{s e}\right\}$, is such that informed traders place only large orders (or refrain from trading if they observe the bad signal and do not own the asset). Thus, small trades are not information-based and do not affect the public belief about the true asset value, while the information content of block trades is very strong.

This implies that the equilibrium price for small orders is given by: ${ }^{6}$

$$
B_{S}^{s e}=A_{S}^{s e}=E[\widetilde{V}]=\pi
$$

and the equilibrium prices for large orders are given by:

$$
\begin{aligned}
& B_{L}^{s e}=\frac{\gamma \pi}{\gamma \pi+(\gamma+\mu \delta)(1-\pi)}=\frac{\gamma \pi}{\gamma+\mu \delta(1-\pi)} \\
& A_{L}^{s e}=\frac{(\gamma+\mu) \pi}{(\gamma+\mu) \pi+\gamma(1-\pi)}=\frac{(\gamma+\mu) \pi}{\mu \pi+\gamma},
\end{aligned}
$$

where $\gamma+\mu \delta$ and $\gamma$ are the probabilities of a block sell order conditional on $\widetilde{V}=0$ and $\widetilde{V}=1$, respectively, and $\gamma+\mu$ and $\gamma$ are the probabilities of a block buy order conditional on $\widetilde{V}=1$ and $\widetilde{V}=0$, respectively. Notice that because of short selling constraints, information-motivated purchases are more likely than information-motivated sells.

The separating equilibrium arises if, given the price schedule $P^{s e}$, informed traders prefer to trade only the large quantity. This occurs when the profit due to the larger quantity exceeds the better price available for small trades, that is when:

$$
\Pi_{\theta, L}^{s e}(\pi) Q_{L} \geq \Pi_{\theta S}^{s e}(\pi) Q_{S},
$$

where $\prod_{\underline{\theta} L}^{s e}(\pi) \equiv B_{L}^{s e}$ and $\prod_{\bar{\theta}}^{s e}(\pi) \equiv 1-A_{L}^{s e}$ are the separating marginal profits of an institutional trader when the final asset value is low and high, respectively, and $\Pi_{\underline{\theta} L} \equiv$ $E[\widetilde{V}]$ and $\Pi_{\bar{\theta} L} \equiv 1-E[\widetilde{V}]$ represent the deviation marginal profits, that is, the marginal profits of an institutional trader who deviates from the "separating" strategy when the final asset value is low and high, respectively. ${ }^{7}$ Rearranging terms and substituting the price schedule $P^{s e}$, condition (2) becomes

$$
\frac{Q_{L}}{Q_{S}} \geq \frac{\pi}{B_{L}^{s e}}=1+f_{\underline{\theta}}(\pi),
$$

with $f_{\underline{\theta}}(\pi) \equiv(1-\pi) \mu \delta / \gamma$, for the bid side of the market, and

$$
\frac{Q_{L}}{Q_{S}} \geq \frac{1-\pi}{1-A_{L}^{s e}}=1+f_{\bar{\theta}}(\pi),
$$

with $f_{\bar{\theta}}(\pi) \equiv \pi \mu / \gamma$, for the ask side of the market. The left side of conditions (3) and (4) represents the market width. For the separating equilibrium to prevail, it has to be larger than the ratio between the deviation and the separating marginal profits on each side of the market.

Conditions (3) and (4) point out that (i) the ratio between deviation and separating marginal profits of an institutional seller reduces when the public belief increases and, then, on the bid side the separating equilibrium is more likely to arise in bull rather than bear markets $\left(f_{\underline{\theta}}(\pi)\right.$ is, indeed, decreasing in $\left.\pi\right)$, whilst (ii) the ratio between deviation

\footnotetext{
${ }^{6}$ To simplify notation hereafter we will omit the $t$ subscript.

${ }^{7}$ Recall that $E[\widetilde{V} \mid \underline{\theta}]=0$ and $E[\widetilde{V} \mid \bar{\theta}]=1$.
} 
and separating marginal profits of an institutional buyer reduces when the public belief decreases and, then, on the ask side it is more likely to arise in bear rather than in bull markets $\left(f_{\bar{\theta}}(\pi)\right.$ is, indeed, increasing in $\pi$ ) (see figure I). To gain some intuition, consider the ask side of the market. The difference between the profit from buying the large and the small quantity can be written as follows

$$
\left(1-A_{L}^{s e}\right)\left(Q_{L}-Q_{S}\right)-\left(A_{L}^{s e}-\pi\right) Q_{S}
$$

The first term represents the separating gain due to the greater quantity of asset bought and the second term is the loss due to the higher price paid to purchase the first $Q_{S}$ units of the asset. An institutional trader observing the good signal chooses to buy large with probability 1 if this difference is positive. Notice that an increase in the public belief affects both those components of the difference between the profit from buying the large and the small quantity. The effect on the separating gain is negative since the ask price is increasing in $\pi$. The effect on the loss due to the higher price paid to purchase the first $Q_{S}$ units of the asset is ambiguous and depends on the magnitude of $\pi$. Indeed, when $\pi$ is 0 or 1 the distance between $A_{L}^{s e}$ and $\pi$ is zero, whilst it grows as the uncertainty in the market increases (i, e., $\pi$ tends to $1 / 2$ ). This implies that the impact of an increase in the public belief on $\left(A_{L}^{s e}-\pi\right)$ is positive for low values of $\pi$ and becomes negative as $\pi$ grows enough. Since (5) is positive if and only if condition (4) is satisfied, we can conclude that the first effect dominates the second one $\left(\left(A_{L}^{s e}-\pi\right) /\left(1-A_{L}^{s e}\right)\right.$ is, indeed, equal to $\left.f_{\bar{\theta}}(\pi)\right)$.

To conclude, it is also worth noting that the more short selling constraints are severe (lower $\delta$ ), the more the separating equilibrium is likely in the bid side of the market. Indeed, the adverse selection problem on the bid side of the market is less severe when $\delta$ is low, since the probability of an information-motivated sell order is lower. As a consequence, the bid price for the large quantity is nearer to bid price for the small quantity and the incentive to sell a large quantity for an institutional seller is higher (see figure I in the Appendix).

If conditions (3) and (4) are not satisfied on either side of the market, then there can be no separating equilibrium on that side of the market and there will be a pooling equilibrium. In a pooling equilibrium there is a positive probability of the informed trading in both large and small quantities. More precisely, an institutional trader plays a mixed strategy $\sigma_{\underline{\theta}} \equiv\left\{\sigma_{\underline{\theta}, S} ; \sigma_{\underline{\theta}, L}\right\}$ defined on the simplex $\Delta\left(S Q_{S}, S Q_{L}\right)$, if he observes $\underline{\theta}$ and the asset is in its portfolio. He plays the mixed strategy $\sigma_{\bar{\theta}} \equiv\left\{\sigma_{\bar{\theta}, S} ; \sigma_{\bar{\theta}, L}\right\}$ defined on the simplex $\Delta\left(B Q_{S}, B Q_{L}\right)$ if he observes $\bar{\theta}$.

For any $\sigma_{\underline{\theta}}$ and $\sigma_{\bar{\theta}}$, the competitive prices are given by

$$
\begin{aligned}
B_{i}^{p e} & =\frac{\gamma \pi}{\gamma+\mu \delta \sigma_{\underline{\theta}, i}(1-\pi)} \\
A_{i}^{p e} & =\frac{\left(\gamma+\mu \sigma_{\bar{\theta}, i}\right) \pi}{\mu \sigma_{\bar{\theta}, i} \pi+\gamma}
\end{aligned}
$$

for all $i \in\{S, L\}$. For the competitive price schedule $P^{p e}=\left\{B_{L}^{p e}, B_{S}^{p e}, A_{S}^{p e}, A_{L}^{p e}\right\}$ in the pooling equilibrium, informed traders must be indifferent between trading the block or the small quantity. This condition requires

$$
\begin{aligned}
\left(B_{L}^{p e}-E[\widetilde{V} \mid \underline{\theta}]\right) Q_{L} & =\left(B_{S}^{p e}-E[\widetilde{V} \mid \underline{\theta}]\right) Q_{S} \\
\left(E[\widetilde{V} \mid \bar{\theta}]-A_{L}^{p e}\right) Q_{L} & =\left(E[\widetilde{V} \mid \bar{\theta}]-A_{S}^{p e}\right) Q_{S}
\end{aligned}
$$


It is easy to see that conditions (7) and (8) can be satisfied only if the price schedule is such that $B_{L}^{p e} \leq B_{S}^{p e}$ and $A_{L}^{p e} \geq A_{S}^{p e}$. This, in turn, implies that institutional traders are more likely to place the large than the small order.

The pooling equilibrium prevails if condition (2) is not satisfied, that is if the relative distance between deviation and separating profits is larger than the market width. From the previous paragraph we know that private signals are more valuable when they indicate the opposite asset value with respect to the public belief. More precisely, if the liquidation asset value is low, the distance between deviation and separating profits of institutional traders (sellers) is larger when the public belief (and, then, the bid price) is higher, whilst if the liquidation asset value is high, the distance between deviation and separating profits of institutional traders (buyers) is larger when the public belief (and, then, the ask price) is lower. As a consequence, informed sellers are more prone to separate themselves from small liquidity traders in bullish markets and the probability of a large information-based sale, $\sigma_{\underline{\theta}, L}$, is increasing in $\pi$, whilst institutional buyers are more prone to separate themselves from small liquidity traders in bearish markets and the probability of a large information-based purchase, $\sigma_{\bar{\theta}, L}$, is decreasing in $\pi$.

\section{The price impact of block trades}

In our model, the price impact of a trade is the change in the public belief about the liquidation asset value due to that trade. Since institutional traders never sell when observing the good signal and never buy when observing the bad signal, the price impact of a sell is always negative and the price impact of a buy is always positive. The magnitude of the price impact of a trade depends both on the trade's information content and on the weight the market maker attaches to this information.

The information content of a trade is related to its likelihood ratio, that is, the ratio between the probability of the trade conditional to $\widetilde{V}=V$ and the probability of the order conditional to $\widetilde{V}=\bar{V}$. If a trade is totally uninformative about the true asset value then its likelihood ratio is equal to 1 ; the more informative the trade is, the more its likelihood ratio differs from 1 . More specifically, the more informative is a block sale, the more its likelihood ratio is higher than 1 and the more informative is a a block purchase, the more its likelihood ratio is lower than 1 . So, we can define the information content of a block sale as its likelihood ratio and the information content of a block purchase as the reciprocal of its likelihood ratio.

The weight the market maker attaches to the information content of a trade is related to the uncertainty about the assetÕs fundamental value. When in the market there is high uncertainty about the true asset value (that is, when $\pi$ is sufficiently far from 0 and 1 ), then the market maker attaches high weight to the trades' information content. But, when the public belief converges to the low or to the high asset value, then the importance of the trades' information content is lower.

Given the unconditional public belief $\pi$, the price impact measure of a block sale is:

$$
\Delta S(\pi) \equiv\left|B_{L}-\pi\right|=\frac{\pi(1-\pi)\left(\lambda_{S}(\pi)-1\right)}{\pi+(1-\pi) \lambda_{S}(\pi)},
$$

where $\lambda_{S}(\pi) \equiv \operatorname{Pr}\left(S Q_{L} \mid \pi, \underline{V}\right) \backslash \operatorname{Pr}\left(S Q_{L} \mid \pi, \bar{V}\right)$ is the trade's information content, condi- 
tional on the public belief $\pi$, and the price impact measure of a block purchase is:

$$
\Delta B(\pi) \equiv\left|A_{L}-\pi\right|=\frac{\pi(1-\pi)\left(\lambda_{B}(\pi)-1\right)}{\pi \lambda_{B}(\pi)+(1-\pi)},
$$

where $\lambda_{B}(\pi) \equiv \operatorname{Pr}\left(B Q_{L} \mid \pi, \bar{V}\right) \backslash \operatorname{Pr}\left(B Q_{L} \mid \pi, \underline{V}\right)$ is the trade's information content, conditional on $\pi$. Notice that, on both sides of the market, the price impact of a block trade is increasing in its information content, and that both $\Delta S(\pi)$ and $\Delta B(\pi)$ are zero when $\pi$ is equal to 0 or 1.

Let the price impact asymmetry expression of the asset for block trades to be defined as:

$$
J(\pi) \equiv \Delta B(\pi)-\Delta S(\pi) .
$$

$J_{i}(\pi)$ is larger than, equal to, or lower than 0 if and only if the price impact of a block purchase is, respectively, larger than, equal to, or lower than the price impact of a block sale.

Proposition 1. If the market width is large enough and the short selling constraints are significant then the price impact of a block purchase is always larger than the price impact of a block sale.

When the market width is high (i.e., $Q_{L} / Q_{S}$ is large), the separating equilibrium arises for all public beliefs and the information content of trades is $\lambda_{S}(\pi)=(\gamma+\mu \delta) / \gamma \equiv \lambda_{S}$ on the bid side and $\lambda_{B}(\pi)=(\gamma+\mu) / \gamma \equiv \lambda_{B}$ on the ask side of the market. In the absence of short selling constraints, $\lambda_{S}=\lambda_{B}$ and the sign of $J(\pi)$ will depend on the public belief. However, if short selling constraints are severe, i.e., $\delta$ is fairly small, sell orders coming from institutional traders are quite unlikely. As a consequence, block sales have small information content (lower than block purchases) and, then, their price impact will be lower than that of purchases. With low market width this result may not be true. Indeed, if $Q_{L} / Q_{S}$ is low, the ask side of a bullish market could be characterized by a pooling equilibrium, whilst the bid side by the separating equilibrium (see conditions (4) and (3) and figure I). In this case, the probability that a trader observing the good signal buys the asset $\left(\mu \sigma_{\bar{\theta}}, L\right)$ could be lower than the probability that a trader observing the bad signal sells the asset $(\mu \delta)$, despite the short selling constraints. This would imply that block sales have higher information content than block purchases and, then, their price impact would be greater than that of purchases.

The public belief about an asset value captures the equilibrium price prior to a trade. Recall that a bullish market is characterized by $\pi>1 / 2$ and a bearish market by $\pi<1 / 2$. This leads to the following result.

Proposition 2. The price impact asymmetry expression is higher in a bear market than in a bull market.

The rationale for the result in Proposition 2 is the following. The equilibrium bid and ask prices for block trades can be viewed as the weighted averages between the public belief and the asset assessment of institutional sellers or buyers. In a bull market, the public belief is nearer to the asset assessment of institutional buyers (1) than to the asset assessment of institutional sellers (0), whilst the opposite is true in a bear market. As a consequence, all other things being equal, in a bull market the price impact of a block purchase (i.e., 
the distance between the ask price and the public belief) is lower than that of a block sale (i.e., the distance between the public belief and the bid price), whilst in a bear market the price impact of a block purchase is larger. This issue is amplified by the fact that in a bull market, the information content of block purchases cannot exceed that of block sales, and in a bear market, the information content of block sales cannot exceed that of block purchases. Indeed, the profit of traders observing a signal contrary to the price path, that is, a good signal in a bear market or a bad signal in a bull market, is larger and induces them to be more aggressive, that is, to trade the large quantity with higher probability.

\section{Data and Method}

The theory developed in the previous section implies that the asymmetry in the permanent price impact of block trades (ie. that the permanent price impact of buys is greater than sales) is greater in a bear market than in a bull market. Following Chiyachantana et. al (2004), the bull market period examined in this study extends from 1 January to 31 December 1997, while the bear market period extends over the first quarter of 2001. The dataset available for this study is similar to the dataset examined by Holthausen et. al (1990) in that it is trade and quote data. In contrast, Chiyachantana et.al (2004) examined packages of trades executed by single institutions. We begin the analysis by re-documenting the asymmetry in the price impact of a sample of block trades across the entire sample period (both bull and bear) using a research method similar to Hothausen et.al (1990), but updated to take into account recent developments in research design and peculiarities of the sample periods we examine.

We consider all trades executed in stocks in the S\&P 500 traded on the New York Stock Exchange over the bull and bear market sample period between 9.30am and 4.00pm. The transaction data used in this study was sourced from Thompson Reuters and is managed and distributed by the Securities Industry Research Centre of Asia Pacific.

Holthausen et.al (1990), use a tick-rule to classify trades into buyer-initiated (upticks) and seller-initiated (downticks), which has been demonstrated to misclassify trades. Consequently, we follow Ellis, Michaely and O'Hara (2000) who use quote based rule to classify all trades as buyer and seller. Specifically, if a trade occurs at the contemporaneous ask price, the trade is classified as buyer-initiated. Conversely, if the trade is executed at the contemporaneous bid price, then the trade is classified as seller-initiated. Trades which execute at neither the bid nor ask price are classified using the tick test. That is, if the last price change was an uptick, the trade is buyer-initiated, and if the last price change was a downtick, the trade is seller-initiated. Bessembinder (2003) argues the merits of the Ellis et. al (2000) classification method vis-á-vis the Lee and Ready (1991) quote method, and tick test for NYSE trade and quote data.

After partitioning trades into buyer or seller initiated, we identify a sample of ÒblockÓ trades. We rely on Holthausen et.al. (1990) definition and sample the 50 largest blocks by trade direction for each stock over each sample period examined (ie. bull market period, bear market period and both combined bull and bear market periods).

The price effects of block trades are measured as follows:

$$
\text { Temporary Price Impact }=\ln \left(\frac{P_{\text {block }}}{P_{\text {post }}}\right)
$$




$$
\begin{aligned}
\text { Permanent Price Impact } & =\ln \left(\frac{P_{\text {post }}}{P_{\text {prior }}}\right) \\
\text { Total Price Impact } & =\ln \left(\frac{P_{\text {block }}}{P_{\text {prior }}}\right)
\end{aligned}
$$

where $P_{\text {prior }}$ is the equilibrium market price prior to the block transaction, and $P_{\text {post }}$ is the equilibrium price after a block trade has been executed. $P_{\text {block }}$ represents the price of the block trade.

In this study two proxies are utilised for equilibrium prices prior and post block trade execution. Following Chiyachantana et al (2004) closing prices on the day before a trade is executed is used as a proxy for $P_{\text {prior }}$, where the closing price is the price of the last trade before $4.00 \mathrm{pm}$. For analysis of price effects measured intraday as in Holthausen et al (1990), the trade price immediately preceding the block is used to proxy $P_{\text {prior }}$, while the transaction price five minutes after the block trade are used to proxy for $P_{\text {post }}$ respectively. Rather than sample prices in transaction time as in Holthausen et. al (1990) (eg. the price of trade $t+5$ relative to the block trade), we sample prices in calendar time (ie. 5 minutes after the block trade has occurred). We do this, as we find that there are large differences in the time between trades during the bull and bear market periods examined which can effect results.

For analysis of price effects using intraday data, we are able to implement a control which enables us to isolate the price impact caused by the larger trade size. Specifically, the price effects of block trades are compared to the price effects of a sample of non-block trades. Following Holtahusen et. al (1990) we match each block transaction with a 100share transaction of the same trade direction (i.e. buyer or seller initiated) by searching prior to the block trade -16 before the block. The difference between the price impacts of block and nonblock trades is referred to as the net effect.

\section{Results}

Table I in the Appendix documents descriptive statistics for the initial sample of block transactions examined in this study. The table documents that there are 19,000 buy and sell transactions sampled across the full sample period. The average trade size of purchases is approximately $\$ 4.9$ million or 120,300 shares while the average size of sales is $\$ 5.6$ million or 139,204 shares $Đ$ confirming that they are block trades by any definition, and larger than the NYSE definition of a block trade (10,000 shares). Similar conclusions can be drawn from the samples drawn separately from bull and bear markets, though there is evidence that trades sampled were larger during the (earlier) bull market period than the bear market period, both in shares as well as in dollar value terms.

Hotlhausen et.al (1990) document that the total price effect of block buys is predominately permanent and there is very little evidence of a temporary price impact (or reversal following the trade), while the total price effect of block sales contains a temporary component which is significantly reversed following the block trade. Table in the Appendix documents total, temporary and permanent price effects for block purchases and sales examined in this study across the entire combined sample period (ie. in both bull and bear markets). Our sample of block trades behaves very similarly to Holthasen et. al (1990). Specifically, there is no evidence of a price reversal following block purchases. The temporary price effect following block purchases is also negative, implying the price continues 
moving upwards after a block purchase, even after controlling for normal market movements following purchases using the nonblock sample. Further, the temporary price effect following block sales is also negative, implying the price rebounds after block sales, even after controlling for normal market movements following sales using the nonblock sample. Similarly to Holthausen et. al (1990) we conclude that the total price impact of block trades is predominately permanent while the total price impact following block sales contains both a temporary and permanent component. A comparison of the permanent price impact of block buys and sales confirms that both the raw and net permanent effects of block buys are greater than block sales. A t test which examines whether the difference in the magnitude of the permanent price impact of block buys is greater than sales is reported in Panel C, and confirms that the difference is significant at conventional levels of significance. We conclude that the price impact asymmetry documented by Holthausen et. al (1990) and others is also present in our (entire) sample.

Chiyachantana et. al (2004) examine the total price impact of institutional trades during the bull and bear market periods examined in this study. For a sample of transactions executed on the NYSE during these sample period they find as follows:

"The patterns of price impact of institutional trading in US stocks (not reported in Table III) are just as striking; 0.59 percent for purchases and 0.21 percent of sells in 1997 in contrast to 0.16 percent for purchases and 0.83 percent for sells in 2001.'[p. 884]

Hence, they find that the total price impact of buys is greater than sells in bull markets, while the total price impact of sells is greater than buys in a bear market. We do not have trade packages available to us, just individual trades, hence we re-examine the Chiyachantana et.al (2004) hypothesis during the bull and bear sample periods to determine whether the results documented by Chiyanchantana et.al (2004) hold despite the methodological and sample differences between this study and theirs.

Table III in the Appendix reports the total impact of buy and sell trades during bull and bear market sample periods. Recall that similarly to Chiyachantana et. al (2004) the benchmark price is the closing price on the day prior to the date on which a trade is executed. While the magnitudes are somewhat smaller, the patterns of the price impact of trades are just as striking as those documented by Chiyachantana et. al (2004): for bull markets, the mean total price impact of buys (0.4292) is greater in magnitude than sells (-0.3178), and a t test reported at the bottom of the table confirms that the magnitude of the differences are statistically significant at conventional levels. In contrast, for bear markets, the magnitude of the total price impact of sells (-0.4260) is greater than buys (0.3349), and again a $t$ test reported at the bottom of the table confirms that the magnitude of the differences are statistically significant at conventional levels. Hence, the results documented by Chiyachantana et. al (2004) are robust to any methodological differences. Having established that our sample behaves entirely like those in previous research in that (1) there is evidence of an asymmetry in the price impact of trades across the entire sample period, and (2) the total price impact of buys is greater than sells in a bull market while the reverse is true in a bear market, we now move to tests of our theory. Specifically, we examine the permanent price impact of buy and sell trades in bull and bear markets. The results of this analysis are reported in Table IV in the Appendix.

Table IV documents that the permanent price impact of purchases is greater than the permanent price impact of sales in both bull and bear markets. However, consistent with our theory, the differences between the two are greater in a bear market (4 basis points) 
than in a bull market ( 2 basis points). Furthermore, a significance test is reported in Panel $\mathrm{B}$, which confirms that the differences in the price impact of buys and sells across the bull and bear market periods are statistically significant at conventional levels of significance. This evidence is consistent with our theoretical proposition that buys are relatively more informative than sells in bear markets relative to bull markets.

Following Chiyachantana et. al (2004) we examine the asymmetry in price impact of block trades for each of the months sampled. Figure II in the Appendix documents the differences between the average permanent price impact of buy and sell trades for each month in our sample drawn from the bear market period (1997) and bull market period (2001). The diagram illustrates that during the months drawn from the bear market that the price impact of buys over sells is generally greater (positive) and higher than in the months drawn from the bull market period. Note that there are 3 months which represent an exception to this during the bull market period, however in two of those months (March and August 1997) the average return on the market was negative and hence in those months in can be argued that the market was in fact bearish. On the balance, there is reasonably strong evidence consistent with our hypothesis that buy trades have a greater permanent impact on prices than sell trades during bear markets.

\section{Summary and Conclusion}

Previous research has documented a 'puzzle 'in the price impact of block trades, finding that the information effects of block buy trades is generally greater than the information effects of block sell trades. Another strand of literature has examined the total price effects of buy and sell transactions in bull and bear markets. This paper extends this literature by examining the information effects of block buy trades and block sell trades in bull and bear market conditions. We develop a theoretical model which predicts that this difference in the magnitude of the information conveyed by buy trades over sell trades is greater in bear markets than bull markets, consistent with the (almost counter-intuitive) proposition that buy trades are relatively more informed in bear markets. Using a sample of trades executed on the NYSE in bull and bear market period identified in previous research, we find evidence consistent with our theoretical proposition. 


\section{Appendix A. Proofs}

\section{Proof of Proposition 1}

First consider conditions (3) and (4). Since $\max _{\pi} f_{\underline{\theta}}(\cdot)=\mu \delta / \gamma$ and $\max _{\pi} f_{\bar{\theta}}(\cdot)=$ $\mu / \gamma>\mu \delta / \gamma$, from conditions (3) and (4) it follows that if $Q_{L} / Q_{S} \geq \mu / \gamma$ then the separating equilibrium prevails on both sides of the market, regardless of public belief.

In the separating equilibrium, $\lambda_{S}(\pi)=(\gamma+\mu \delta) / \gamma \equiv \lambda_{S}$ and $\lambda_{B}(\pi)=(\gamma+\mu) / \gamma \equiv$ $\lambda_{B}$. By substituting $\lambda_{S}$ and $\lambda_{B}$ in (9) and (10), and then (9) and (10) in (11) and rearranging terms gives

$$
J(\pi)=\frac{\pi(1-\pi) \mu}{(\pi \mu+\gamma)(\gamma+(1-\pi) \mu \delta)} \phi(\pi ; \delta),
$$

where $\phi(\pi ; \delta)=\gamma(1-\delta)+(1-2 \pi) \mu \delta$. Since $\min _{\pi} \phi(\cdot ; \delta)=\gamma(1-\delta)-\mu \delta$, we can conclude that $\phi(\pi ; \delta)$ is positive for all $\pi$ iff $\delta$ is lower than $\gamma /(\gamma+\mu)$.

\section{Proof of Proposition 2}

Assume $\pi \geq 1 / 2$ and define $\Delta J(\pi) \equiv J(\pi)-J(1-\pi)$ as the difference between the price impact asymmetry in the bullish and in the bearish market. ${ }^{8}$ Notice that:

$$
\begin{aligned}
& \Delta J(\pi) \equiv(\Delta B(\pi)-\Delta S(\pi))-(\Delta B(1-\pi)-\Delta S(1-\pi))= \\
& \quad=(\Delta B(\pi)-\Delta B(1-\pi))-(\Delta S(\pi)-\Delta S(1-\pi)) .
\end{aligned}
$$

We will prove the proposition by showing that this difference is negative if and only if $\pi \geq 1 / 2$, both in the separating equilibrium (Step 1) and in the pooling equilibrium (Steps 2 and 3$)$.

Step 1: If the separating equilibrium prevails on both sides of the market both when $E[\widetilde{V}]=\pi$ and when $E[\widetilde{V}]=1-\pi$, then $\Delta J(\pi) \leq 0$ if and only if $\pi \geq 1 / 2$.

Assume $\lambda_{S}(\pi)=\lambda_{S}$ and $\lambda_{B}(\pi)=\lambda_{B}$. Substituting (9) in $(\Delta S(\pi)-\Delta S(1-\pi))$ and rearranging terms gives

$$
\Delta S(\pi)-\Delta S(1-\pi)=-\frac{(1-2 \pi) \pi(1-\pi)\left(\lambda_{S}-1\right)^{2}}{\left(\pi \lambda_{S}+(1-\pi)\right)\left(\pi+(1-\pi) \lambda_{S}\right)},
$$

that is positive since $\pi \geq 1 / 2$ by assumption. Similarly, substituting (10) in $(\Delta B(\pi)-$ $\Delta B(1-\pi))$ and rearranging terms gives

$$
\Delta B(\pi)-\Delta B(1-\pi)=\frac{(1-2 \pi) \pi(1-\pi)\left(\lambda_{B}-1\right)^{2}}{\left(\pi \lambda_{B}+(1-\pi)\right)\left(\pi+(1-\pi) \lambda_{B}\right)},
$$

that is negative since $\pi \geq 1 / 2$. By combining (13) and (14) with (12) we can conclude that $\Delta J(\pi)$ is negative.

\footnotetext{
${ }^{8}$ Recall that a bullish market is characterized by $\pi>1 \backslash 2$ and a bearish market by $\pi<1 \backslash 2$.
} 
Step 2: If $\lambda_{S}(\pi) \geq \lambda_{S}(1-\pi)$ and $\lambda_{B}(\pi) \leq \lambda_{B}(1-\pi)$, then $\Delta J(\pi) \leq 0$ if and only if $\pi \geq 1 / 2$.

Assume that $\lambda_{S}(\pi) \geq \lambda_{S}(1-\pi)$ and $\lambda_{B}(\pi) \leq \lambda_{B}(1-\pi)$. From the previous step, we know that, if $\lambda_{S}(\pi)=\lambda_{S}(1-\pi)$ and $\lambda_{B}(\pi)=\lambda_{B}(1-\pi)$, then $\Delta S(\pi)-\Delta S(1-$ $\pi) \geq 0$ and $\Delta B(\pi)-\Delta B(1-\pi) \leq 0$ for all $\pi \geq 1 / 2$. Deriving (9) with respect to $\lambda_{S}$ and rearranging terms gives

$$
\frac{\partial \Delta S\left(\pi ; \lambda_{S}\right)}{\partial \lambda_{S}}=-\frac{(1-\pi) \pi}{\left(\pi+(1-\pi) \lambda_{S}\right)^{2}},
$$

that is positive for all $\pi$. This implies that for all $1<\lambda_{S}^{\prime \prime} \leq \lambda_{S}^{\prime}$

$$
\Delta S\left(\pi ; \lambda_{S}^{\prime}\right)-\Delta S\left(1-\pi ; \lambda_{S}^{\prime \prime}\right) \geq \Delta S\left(\pi ; \lambda_{S}^{\prime}\right)-\Delta S\left(1-\pi ; \lambda_{S}^{\prime}\right)
$$

Deriving (10) with respect to $\lambda_{B}$ and rearranging terms gives

$$
\frac{\partial \Delta B\left(\pi ; \lambda_{B}\right)}{\partial \lambda_{B}}=\frac{(1-\pi) \pi}{\left(\pi \lambda_{B}+(1-\pi)\right)^{2}},
$$

that is positive for all $\pi$. This implies that for all $\lambda_{B}^{\prime \prime} \geq \lambda_{B}^{\prime}>1$

$$
\Delta B\left(\pi ; \lambda_{B}^{\prime}\right)-\Delta B\left(1-\pi ; \lambda_{B}^{\prime \prime}\right) \leq \Delta B\left(\pi ; \lambda_{B}^{\prime}\right)-\Delta B\left(1-\pi ; \lambda_{B}^{\prime}\right) .
$$

By combining (15) and (16) with (12) we can conclude that

$$
\Delta J(\pi)\left|\begin{array}{l}
\lambda_{S}(\pi) \geq \lambda_{S}(1-\pi) \\
\lambda_{B}(\pi) \leq \lambda_{B}(1-\pi)
\end{array} \leq \Delta J(\pi)\right| \begin{aligned}
& \lambda_{S}(\pi)=\lambda_{S}(1-\pi) \\
& \lambda_{B}(\pi)=\lambda_{B}(1-\pi)
\end{aligned},
$$

that is negative since $\pi \geq 1 / 2$ by assumption.

Step 3: In the equilibrium, the information content of block sell orders is such that $\lambda_{S}(\pi) \geq \lambda_{S}(1-\pi)$, and the information content of block sell orders is such that $\lambda_{B}(\pi) \leq \lambda_{B}(1-\pi)$ for all $\pi \geq 1 / 2$.

We will prove the proposition for the ask side of the market. The proof for the bid side is symmetric and will be omitted. From the proof of Proposition 1 we already know that If the separating equilibrium prevails on the ask side of the market both when $E[\widetilde{V}]=\pi$ and when $E[\widetilde{V}]=1-\pi$, then $\lambda_{B}(\pi)=\lambda_{B}(1-\pi)=\lambda_{B}$. The function $f_{\bar{\theta}}(\cdot)$ in condition (4) is increasing in the public belief $\left(\partial f_{\bar{\theta}}(\cdot) / \partial \pi=\mu / \gamma>0\right)$. From condition (4), it follows that if the separating equilibrium arises when the public belief is $\pi$, then it arises for all public belief lower than $\pi$. As a consequence, if $\lambda_{B}(\pi)=\lambda_{B}$ then $\lambda_{B}(1-\pi)=\lambda_{B}$, as well. ${ }^{9}$ On the other hand, if when the public belief is $\pi$ a pooling equilibrium arises, that is $\lambda_{B}(\pi)<\lambda_{B}$, then when the public belief is $1-\pi$ either a separating or a pooling equilibrium can prevail. The separating equilibrium arises if $Q_{L} / Q_{S} \geq 1+f_{\bar{\theta}}(1-\pi)$, and $\lambda_{B}(1-\pi)=\lambda_{B}>$ $\lambda_{B}(\pi)$. The pooling equilibrium arises if $Q_{L} / Q_{S}<1+f_{\bar{\theta}}(1-\pi)$ and, also in this case, $\lambda_{B}>\lambda_{B}(1-\pi)>\lambda_{B}(\pi)$. Indeed, suppose that also when the public belief is $1-\pi$ condition (4) is not satisfied. Let be $\{(1-\sigma) ; \sigma\}$ the strategy of an institutional buyer, and notice that, for any $\sigma$, the informational content of a block buy is $\lambda_{B Q_{L}}(\sigma)=(\gamma+\mu \sigma) / \gamma$ and that of a small buy is $\lambda_{B Q_{S}}(\sigma)=(\gamma+\mu(1-\sigma)) / \gamma$. Rearranging terms in (6), the ask price for quantity $Q_{i}$, with $i \in\{S, L\}$, is

$$
A_{i}^{p e}=\frac{\lambda_{B Q_{i}}(\sigma) \pi}{\lambda_{B Q_{i}}(\sigma) \pi+(1-\pi)} .
$$

\footnotetext{
${ }^{9}$ We are assuming $\pi \geq 1 / 2$, then $1-\pi \leq \pi$
} 
Substituting $E[\widetilde{V} \mid \bar{\theta}]=1$ and (18) in (8) and rearranging terms gives

$$
\frac{Q_{L}}{Q_{S}}=\frac{\lambda_{B Q_{L}}(\sigma) \pi+(1-\pi)}{\lambda_{B Q_{S}}(\sigma) \pi+(1-\pi)}=f(\pi ; \sigma)
$$

For any $\pi$, the equilibrium strategy $\sigma(\pi)$ has to satisfy condition (19). By Dini's theorem, this implies that

$$
\begin{gathered}
\frac{d \sigma}{d \pi}=-\frac{\partial f(\pi ; \sigma) / \partial \pi}{\partial f(\pi ; \sigma) / \partial \sigma}= \\
=-\frac{\lambda_{B Q_{L}}(\sigma)-\lambda_{B Q_{S}}(\sigma)}{\pi\left[\left(\lambda_{B Q_{S}}(\sigma) \pi+(1-\pi)\right) \partial \lambda_{B Q_{L}}(\sigma) / \partial \sigma-\partial \lambda_{B Q_{S}}(\sigma) / \partial \sigma\right]}<0
\end{gathered}
$$

since $\lambda_{B Q_{L}}(\sigma)>\lambda_{B Q_{S}}(\sigma), \partial \lambda_{B Q_{L}}(\cdot) / \partial \sigma=\mu / \gamma>0$ and $\partial \lambda_{B Q_{S}}(\cdot) / \partial \sigma=$ $-\mu / \gamma<0$. Thus, since $\pi \geq(1-\pi)$ for all $\pi \geq 1 / 2$, we can conclude that if a pooling equilibrium arises when the public belief is $1-\pi$ then $\sigma(1-\pi) \geq \sigma(\pi)$ and $\lambda_{B Q_{L}}(\sigma(1-\pi)) \geq \lambda_{B Q_{L}}(\sigma(\pi))$. 
Appendix B. Table and Figures

Iable I. Descriptive statistics for block transictions and nonblock transactions

This table reports the mean size of transactions examined in this study, being the fifty largest trades, by number of ahares for each of buy and sell trades in: (1) the combined sample period of 1997 and 2001, (2) the bear market of 2001 and (3) the bull market of 1997 . For exch block transaction a nonblock transaction of 100 -shares is identified by searching sirteen trades prior to the block trades on the same trading day and same trade direction. Trades are extegorised into buyer or seller initiated trades using a quote-bused rule. Companies are remored from the smple if fifty block trades are not matched.

\begin{tabular}{|c|c|c|c|c|c|c|}
\hline & \multicolumn{2}{|c|}{1997 and 2001 Sample } & \multicolumn{2}{|c|}{ Bear Markets } & \multicolumn{2}{|c|}{ Bull Markets } \\
\hline & Purchases & Sales & Purchases & Sales & Purchnes. & 5yles \\
\hline & \multicolumn{2}{|c|}{$n=19,000$} & \multicolumn{2}{|c|}{$\mathrm{a}=17,000$} & \multicolumn{2}{|c|}{$n=15,600$} \\
\hline \multicolumn{7}{|l|}{ Block Trade } \\
\hline Size & 120,300 & 139,204 & 84,853 & 90,993 & 97,664 & 114,870 \\
\hline \multicolumn{7}{|l|}{ Block Trade } \\
\hline Value & $4,924,420$ & $5,643,493$ & $3,289,640$ & $3,446,467$ & $4,487,825$ & $5,247,260$ \\
\hline \multicolumn{7}{|l|}{ Nonblock } \\
\hline Trade Value & 4,442 & 4,452 & 4,162 & 4,141 & 5,122 & 5,115 \\
\hline
\end{tabular}


Takh II. Temporary, permanent and total price impact of block transactions in bull or bear markets

This table presents mean and medinn total, temporary and permanent price effects for block trades and matched sample of 100-ghare tranasction (nonblock). Price effects are measured as follow:

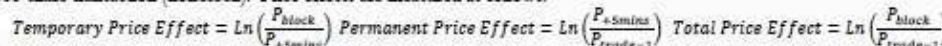

Each block transaction is matched with a 100-share transaction of the same trade direction by searching prior to the trade minus sirteen relative to the block transaction. Trades are desiguated buyer or seller imitated bo the quote rule. The mean difference and test of differebce t-statistics mexaure and teat the difference between the block and matched nonblock samples.

\begin{tabular}{|c|c|c|c|c|c|c|}
\hline & \multicolumn{2}{|c|}{ Temporary Price Effect } & \multicolumn{2}{|c|}{ Permanent Price Effect } & \multicolumn{2}{|c|}{ Total Price Effect } \\
\hline & Block & Nonblock & Block & Nonblock & Block & Noublock \\
\hline \multicolumn{7}{|c|}{ Panel A: Purebases } \\
\hline Mean & $-0.0055 \%$ & $0.0433 \%$ & $0.1870 \%$ & $0.0273 \%$ & $0.1797 \%$ & $0.0687 \%$ \\
\hline Standard Deriation & 0.0042 & 0.0044 & 0.0056 & 0.0046 & 0.0037 & 0.0015 \\
\hline Median & $0,0000 \%$ & $0.0000 \%$ & $0.1100 \%$ & $0.0000 \%$ & $0.0336 \%$ & $0.0000 \%$ \\
\hline m-atatistic & -1.82 & 13.59 & 46.19 & 8.23 & 67.35 & 62.12 \\
\hline $\begin{array}{l}\text { Mean Difference } \\
\text { Test of difference } \\
\text { gtatistic }\end{array}$ & \multicolumn{2}{|c|}{$-0.0488 \%$} & \multicolumn{2}{|c|}{$0.1597 \%$} & \multicolumn{2}{|c|}{$0.1110 \%$} \\
\hline \multicolumn{7}{|c|}{ Panel B: Sales } \\
\hline Mean & $-0.0799 \%$ & $-0.0583 \%$ & $-0.1479 \%$ & $-0.0136 \%$ & $-0.2288 \%$ & $-0.0734 \%$ \\
\hline Standard Deviation & 0.0037 & 0.0040 & 0.0049 & 0.0041 & 0.0038 & 0.0015 \\
\hline Medins & $0.0000 \%$ & $0.0000 \%$ & $-0.0915 \%$ & $0.0000 \%$ & $-0.1416 \%$ & $0.0000 \%$ \\
\hline t-metatistic & -30.10 & -19.99 & -41.41 & -4.53 & -82.64 & -67.22 \\
\hline $\begin{array}{l}\text { Mean Difference } \\
\text { Test of difference r } \\
\text { statistic }\end{array}$ & \multicolumn{2}{|c|}{$-0.0216 \%$} & \multicolumn{2}{|c|}{$-0.1343 \%$} & \multicolumn{2}{|c|}{$-0.1554 \%$} \\
\hline \multicolumn{7}{|c|}{ Panel $C:$ Permanent Price Impact A Armmetry } \\
\hline Block Impact differe & reen buyter, & eller initisted & & & $(t=7.24)$ & \\
\hline Net Block Impact di & between bur & nd seller inis: & rades & & $(t=3.74)$ & \\
\hline
\end{tabular}

venerdì 9 novembre 2012 
Toble III. Total price impact of block transactions in bull and bear markets

The table presents estimates of the total impact costs of block transactions in the bear market of 2001 and the bull market of 1997 . The toral price effeet is defined as follows:

$$
\text { Total Price Effect }=\ln \left(\frac{P_{\text {block }}}{P_{\text {close } \Delta a y-1}}\right)
$$

Mean and median price effects are reported for the fifty largest trades, by number of shares for each trade direction type and compant in: (1) the bear market of 2001 and (2) the bull market of 1997. The mean difference and test of difference fatatistics mearure and test the difference between the block purchase and sale transactions.

\begin{tabular}{|c|c|c|c|c|}
\hline & \multicolumn{2}{|c|}{ Bear Markets } & \multicolumn{2}{|c|}{ Bull Markets } \\
\hline & Purcbases & Sales & Purchase: & Sales \\
\hline Menn & $0.3349 \%$ & $-0.4260^{\%} \%$ & $0.4292 \%$ & $-0.3178 \%$ \\
\hline $\begin{array}{l}\text { Standard } \\
\text { Derriation }\end{array}$ & 0.0318 & 0,0299 & 0.0184 & 0.0197 \\
\hline Median & $0.1282 \%$ & $-0.3629 \%$ & $0.3509 \%$ & $-0.2655 \%$ \\
\hline Fatatistic & 13.75 & -18.59 & 29.22 & -20.10 \\
\hline Observations & 17,000 & 17,000 & 15,600 & 15,600 \\
\hline $\begin{array}{l}\text { Mean difference } \\
\text { Teat of } \\
\text { difference t } \\
\text { statistic }\end{array}$ & -0.0 & & 0.1 & \\
\hline
\end{tabular}

venerdì 9 novembre 2012 
Ioble IV . Permanent price impact of block trades in bull and bear markets

The table presents estimates of the net permanent impaet costs of block transaction in the bear market of first quarter 2001 and the bull maxket of 1997 . The net perminent price impact is defined as the difference in permanent price effect is of block and nonblock transactions (ie 2 matched 100-share transzetion of the same trade direction oceurring sirteen trades prior to the relative to the block transaction. Permanent price effects is messured as follows:

$$
\text { Permanent Price Ef fect }=\operatorname{Ln}\left(\frac{P_{\text {tsmita }}}{P_{\text {trede }-1}}\right)
$$

Net mean and medinn price effects are reported for block purchases and block sales). The menn difference and test of difference Natatistics measure and test the difference between the block purchase and sale transactions. Fanel B testa the proposition thrt the permawent price impact asymmetry is higher in bear markets vis-1-tis bull markets. Panel A: Permanent Price Impact Asymmetry

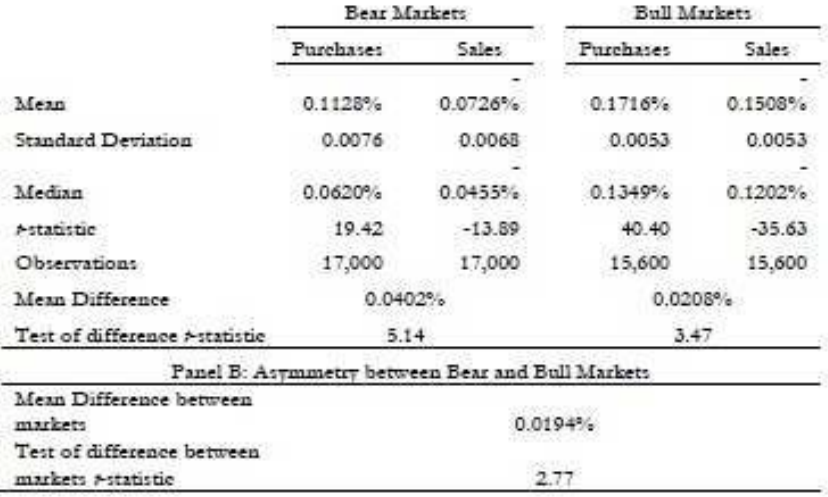

venerdì 9 novembre 2012 
Figure I: $f_{\bar{\theta}}(\pi)+1$ is the ratio between the deviation and the separating profits of an informed buyer, $f_{\theta}(\pi, \delta=1)+1$ and $f_{\theta}(\pi, \delta<1)+1$ are the ratio between the deviation and the separating profits of an informed seller, without $(\delta=1)$ and with $(\delta<1)$ short selling constraints, for any public belief $\pi . \frac{Q_{L}}{Q_{S}}$ represents the market width. On the ask side of the market the separating equilibrium arises only when the public belief is below $\hat{\pi}$, since for all $\pi>\hat{\pi} \quad f_{\bar{\theta}}(\pi)+1$ is larger than $\frac{Q_{L}}{Q_{S}}$. Symmetrically, in the absence of short selling constraints, on the bid side of the market the separating equilibrium arises only when the public belief is above $1-\hat{\pi}$, since for all $\pi<1-\hat{\pi}$ $f_{\underline{\theta}}(\pi, \delta=1)+1$ is larger than $\frac{Q_{L}}{Q_{S}}$. Instead, if short selling constraints are sufficiently severe (that is, $\delta$ small enough), the separating profits are greater and $f_{\underline{\theta}}(\pi, \delta<1)+1$ is lower than $\frac{Q_{L}}{Q_{S}}$ for all $\pi$.

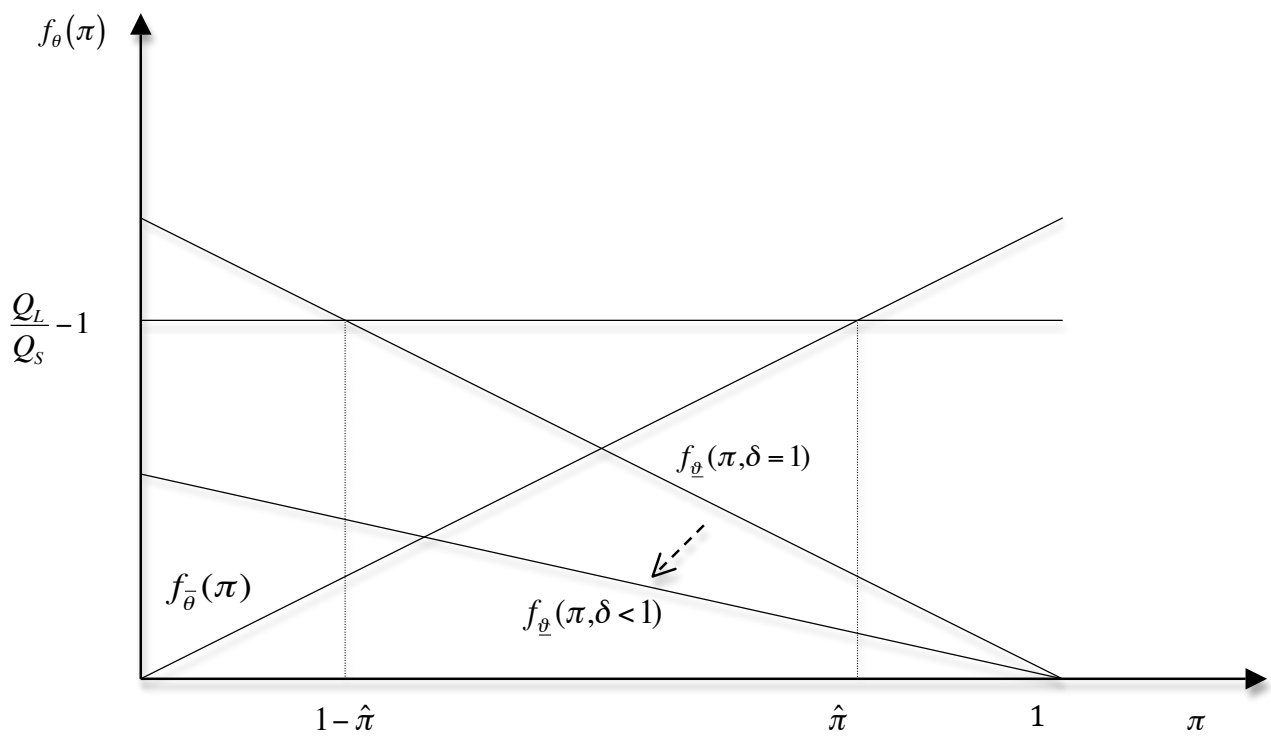




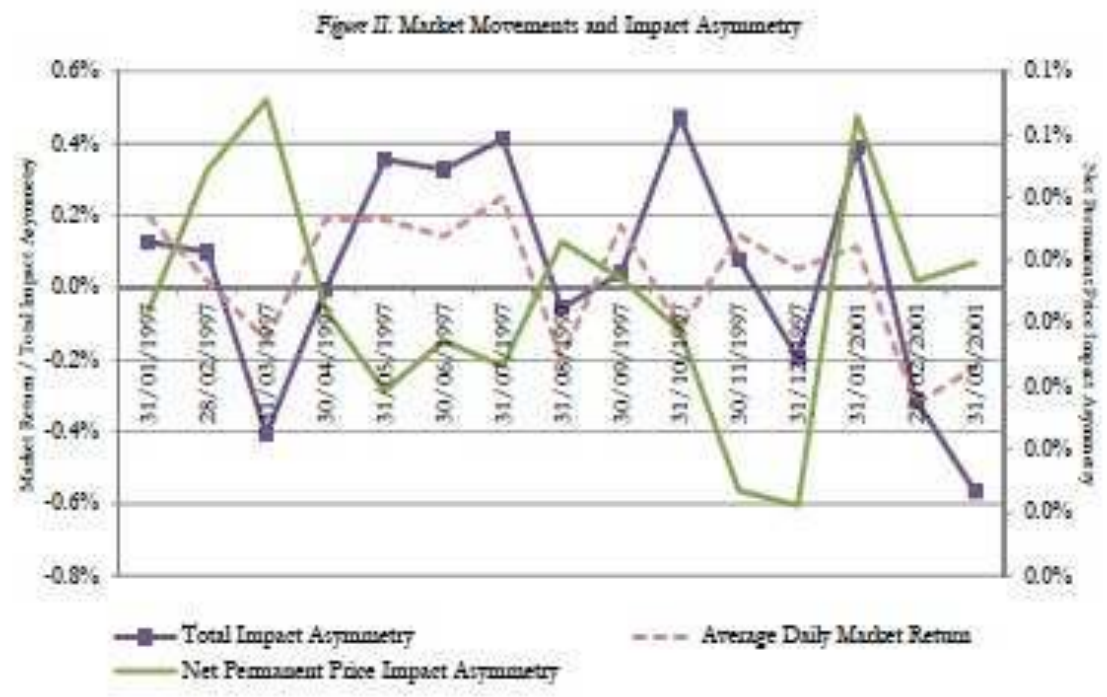

venerdì 9 novembre 2012

\section{References}

[1] Aitken, M.J. and Frino, A. (1996), "Execution costs associated with institutional trades on the Australian Stock Exchange," Pacific-Basin Finance Journal, 4, pp.4558.

[2] Bessembinder, H. (2003), "Issues in Assessing Trade Execution Costs," The Journal of Financial Markets, 6, pp. 233-257.

[3] Chan, L.K.C. and Lakonishok, J. (1993), "Institutional trades and intraday stock price behaviour," Journal of Financial Economics, 33, pp. 173-199.

[4] Chan, L.K.C. and Lakonishok, J. (1995), "The behaviour of stock prices around institutional trades," Journal of Finance, 50(4), pp. 1147-11174.

[5] Chiyachantana, C.N., Jain, P.K., Jiang, C. and Wood, R.A. (2004), "International evidence on institutional trading behaviour and price impact," Journal of Finance, 59, pp. 869-898.

[6] Easley, D.N. and O'Hara, M. (1987), "Price, trade size, and information in securities markets," Journal of Financial Economics, 19, pp. 69-90.

[7] Ellis, K., Michaely, R. and OÕHara, M. (2000), "The accuracy of trade classification rules: Evidence from NASDAQ," Journal of Financial and Quantitative Analysis, 35, pp. 529-552. 
[8] Gemmill, G. (1996), "Transparency and liquidity: A study of block trades in the London Stock Exchange under different publication rules," Journal of Finance, 51, pp.1765-1790.

[9] Holthausen, R., Leftwich, R. and Myers, D. (1987), "The effect of large block transactions on security prices: A cross-sectional analysis," Journal of Financial Economics, 19, pp. 237-268.

[10] Holthausen, R., Leftwich, R. and Myers, D. (1990), "Large-block transactions, the speed of response, and temporary and permanent stock-price effects," Journal of Financial Economics, 26, pp.71-95.

[11] Keim, D.B. and Madhavan, A. (1995), "Anatomy of the trading process: Empirical evidence on the behaviour of institutional traders," Journal of Financial Economics, 37, pp. 371-398.

[12] Keim, D.B. and Madhavan, A. (1996), "The upstairs market for large-block transactions: Analysis and measurement of price effects," Review of Financial Studies, 9, pp. 1-36.

[13] Kraus, A. and Stoll, H. (1972), "Price impacts of block trading on the New York Stock Exchange," Journal of Finance, 27, pp. 569-588.

[14] Lee, C.M.C. and Ready, M.J. (1991), "Inferring trade direction from intraday data," Journal of Finance, 46, pp. 733-746. pp. 45-58.

[15] Saar, G. (2001), "Price impact asymmetry of block trades: An institutional trading explanation," Review of Financial Studies, 14, pp. 1153-1182. 